\title{
CONSTITUINTES QUÍMICOS E ATIVIDADE INSETICIDA DE Miconia ferruginata
}

\author{
Gracielle Oliveira Sabbag Cunha ${ }^{\mathrm{a}}$, Andreia Pereira Matos ${ }^{\mathrm{b}}$, Antonio Rogerio Bernardo ${ }^{\mathrm{b}}$, Antonio Carlos Severo Menezes ${ }^{\mathrm{a}}$, \\ Marcela Carmen de Melo Burger ${ }^{\text {, }}$ Paulo Cezar Vieira ${ }^{\mathrm{b}}$, Moacir Rossi Forim ${ }^{\mathrm{b}}$, João Batista Fernandes ${ }^{\mathrm{b}, *}$ e Maria Fatima \\ das Graças Fernandes da Silva ${ }^{b}$ \\ aUnidade de Ciências Exatas e Tecnológicas, Universidade Estadual de Goiás, 75132-400, Anápolis - GO, Brasil \\ bDepartamento de Química, Universidade Federal de São Carlos, CP 676, 13565-905 São Carlos - SP, Brasil
}

Recebido em 02/04/2017; aceito em 08/08/2017; publicado na web em 02/10/2017

\begin{abstract}
CHEMICAL CONSTITUENTS AND INSECTICIDAL ACTIVITY OF THE Miconia ferruginata. Phytochemical investigation of the leaf extracts from Miconia ferruginata (Melastomataceae) led to the isolation and characterization of the flavonoids 5,6,7-trihydroxy4'-methoxyflavone, 5-hydroxy-7,4'-dimethoxy-8-methylflavone and 5,7,4'-trihydroxy-6,8-dimetilflavone, triterpenes ursolic acid and oleanolic acid, in addition to the steroids $\beta$-sitosterol and stigmasterol. The structures of these compounds were proposed by spectroscopic analysis and comparison with literature data. This paper also describes the insecticidal effects of leaf extracts from $M$. ferruginata to one of the main pests of maize, the fall armyworm (Spodoptera frugiperda). The ingestion bioassays, the leaf extracts showed an elongation of the larval stage of 16.56 days as compared to the control.
\end{abstract}

Keywords: phytochemical studies; flavonoids; insecticidal activity; Spodoptera frugiperda.

\section{INTRODUÇÃO}

A família Melastomataceae, pertencente à ordem Myrtales, é formada por aproximadamente 170 gêneros que incluem cerca de 4500 espécies. ${ }^{1} \mathrm{Na}$ América tropical, esta família está representada por aproximadamente 3000 espécies, distribuídas em 100 gêneros, sendo Miconia o maior gênero da família, com cerca de 1000 espécies que ocorrem desde o sul do México até norte de Argentina e Uruguai. ${ }^{1,2}$ No Brasil estão representadas cerca de 250 espécies. ${ }^{3}$ O gênero Miconia é ainda pouco conhecido com relação ao seu potencial químico. Das espécies até então investigadas foram isoladas benzoquinonas, ${ }^{4}$ triterpenos, ${ }^{5-9}$ flavonoides, ${ }^{10-12}$ compostos fenólicos e taninos. ${ }^{13}$

Poucas são as referências à trabalhos de investigação sobre os metabólitos de Miconia ferruginata. As únicas investigações relacionadas à espécie relatam a presença de flavonoides, saponinas, cumarinas, terpenos e taninos nas folhas da espécie. ${ }^{14,15} \mathrm{Em}$ relação à atividade inseticida do gênero Miconia, estudos anteriores demonstraram os efeitos de extratos de Miconia sp. sobre o fungo Leocoagaricus gongylophorus. ${ }^{16}$ Extratos de M. affinis foram testados sobre os fungos fitopatogênicos (Botryotinia fuckeliana, Magnaporthe oryzae, Phytophtora infestans e Septoria tritici). ${ }^{17}$

A cultura do milho é de suma importância para a economia do Brasil, sendo este o terceiro maior produtor mundial. O uso indiscriminado de inseticidas sintéticos convencionais no controle de pragas do milho causa a contaminação dos recursos hídricos, alimentos, reduzem o número de inimigos naturais e muitas vezes causam resistência dessas pragas a tais inseticidas. $\mathrm{O}$ uso de extratos vegetais e óleos essenciais isolados de plantas é uma estratégica alternativa ao uso de inseticidas sintéticos convencionais. Dentre os insetos pragas mais comuns nos cultivos de milho há a lagarta-do-cartucho do milho, Spodoptera frugiperda (J. E. Smith), podendo causar uma redução de até $34 \%$ na produção de grãos, dependendo do estágio de desenvolvimento da cultura no momento em que ocorre o ataque. ${ }^{18}$ No milho, a lagarta é usualmente controlada com piretróides e organofosforados. ${ }^{19}$ Dessa forma, técnicas alternativas de controle vêm sendo investigadas,

*e-mail: djbf@ufscar.br incluindo-se dentre elas, os inseticidas de origem vegetal. Assim, o presente trabalho teve como objetivo o estudo fitoquímico de extratos de folhas de $M$. ferruginata e a ação biológica destes extratos sobre a lagarta-do-cartucho do milho, S. frugiperda.

\section{PARTE EXPERIMENTAL}

\section{Procedimentos experimentais gerais}

Os espectros de $\mathrm{RMN}{ }^{1} \mathrm{H} \mathrm{e}{ }^{13} \mathrm{C}$ (uni e bidimensionais) foram obtidos em espectrômetro Bruker DRX $400 \mathrm{MHz}$, utilizando-se $\mathrm{CDCl}_{3}$, acetona-d6 ou $\mathrm{CD}_{3} \mathrm{OD}$ como solventes e TMS como padrão interno.

Para identificação dos esteroides foi utilizado cromatógrafo com fase gasosa acoplado ao espectrômetro de massas, marca Shimadzu, modelo QP-5000, com coluna capilar AT-5 MS (30 m x 0,25 mm), utilizando as seguintes condições: temperatura do injetor: $250{ }^{\circ} \mathrm{C}$, gás de arraste: He, temperatura inicial do forno: $150{ }^{\circ} \mathrm{C}$ por $3 \mathrm{~min}$, velocidade de aquecimento a $6{ }^{\circ} \mathrm{C} / \mathrm{min}$ até $280{ }^{\circ} \mathrm{C}$, permanecendo nesta temperatura por $20 \mathrm{~min}$. $\mathrm{O}$ espectro de massas foi obtido por impacto de elétrons a $70 \mathrm{eV}$.

As separações cromatográficas em colunas foram realizadas utilizando-se gel de sílica 60, 70-230, 230-400 mesh e Sephadex LH-20. As análises cromatográficas em camada fina foram realizadas em cromatoplacas de sílica gel $\mathrm{F}_{254}$ sobre placa de alumínio Merck, de $0,2 \mathrm{~mm}$ de espessura, empregando-se como revelador a solução de vanilina/ácido sulfúrico.

\section{Material vegetal}

As folhas de $M$. ferruginata foram coletadas na vegetação do Cerrado da Universidade Estadual de Goiás, Anápolis no dia 10 de setembro de 2008 no início da manhã. Uma exsicata foi depositada no herbário desta universidade, sob o ${ }^{\circ}$ 5794. A identificação foi feita pela Profa. Dra. Mirley Luciene dos Santos.

\section{Obtenção dos extratos das partes vegetais}

As folhas de $M$. ferruginata (1485 g) foram secas em estufa de circulação de ar Marconi Modelo MA-035 a $45{ }^{\circ} \mathrm{C}$ e pulverizadas 
em moinho de facas tipo Croton Marconi Modelo MA-580 Willey. A extração do material moído foi realizada por maceração em etanol $(5 \mathrm{~L})$ por três dias, à temperatura ambiente, seguida pela evaporação do solvente em rotaevaporador Tecnal TE-210, levando à obtenção do extrato etanólico bruto $(405,11 \mathrm{~g})$.

\section{Fracionamento do extrato}

Parte do extrato etanólico bruto de folhas (112,62 g, 27,8\%) foi suspenso em uma mistura EtOH/ $\mathrm{H}_{2} \mathrm{O} 1: 3$ e particionado sucessivamente com hexano, $\mathrm{CH}_{2} \mathrm{Cl}_{2}$ e AcOEt. Foram obtidas 4 frações após evaporação dos solventes sendo: MFFE-H (19,63 g, 4,84 \%; hexano); MFFE-D (0,86 g, 0,21\%; diclorometano); MFFE-A (24,30 g, 6,00\%; acetato de etila) e MFFE-W (65,19 g, 16,09\%, aquosa).

\section{Fracionamento da fração MFFE-D}

A fração MFFE-D (0,86 g) foi fracionada em coluna $(\phi \times \mathrm{h}=3 \times 16 \mathrm{~cm})$ de sílica gel $\left(230\right.$ - 400 mesh), eluída com $\mathrm{CH}_{2} \mathrm{Cl}_{2} /$ acetona $(9,5: 0,5)$ em eluição isocrática. Posteriormente, a fração MFFE-D5 (233 mg) foi refracionada em coluna $(\phi \times \mathrm{h}=3 \times 15 \mathrm{~cm})$ de sílica gel (230 - 400 mesh), eluída com $\mathrm{CH}_{2} \mathrm{Cl}_{2} /$ Acetona (9:1) em eluição isocrática. As subfrações resultantes, MFFE-D5.3 (20,1 mg) e MFFE-D5.7 (18,0 mg) após submissão em coluna de gel de Sephadex LH-20 $(\phi \times \mathrm{h}=2,4$ x $66 \mathrm{~cm}) \mathrm{com} \mathrm{MeOH} / \mathrm{CH}_{2} \mathrm{Cl}_{2}$ (7:3) e $(1: 1)$, respectivamente, forneceram as substâncias $\mathbf{1}(13,5 \mathrm{mg})$ e $\mathbf{2}(1,7 \mathrm{mg})$.

\section{Fracionamento da fração $M F F E-H$}

A fração MFFE-H (2,0 g) foi fracionada em coluna sob vácuo, empacotada com sílica gel (70 - 230 mesh) em funil de placa sinterizada ( $\phi \times \mathrm{h}=7 \times 26 \mathrm{~cm})$, utilizando-se como fases móveis solventes orgânicos de polaridade crescente: hexano; $\mathrm{CH}_{2} \mathrm{Cl}_{2} ; \mathrm{AcOEt}$ e $\mathrm{MeOH}$. Foram obtidas 4 frações após evaporação dos solventes orgânicos: MFFE-HH $=0,05 \mathrm{~g}$; MFHE-HD $=0,53 \mathrm{~g}$; MFFE-HA $=0,68 \mathrm{~g}$ e MFFE-HM $=0,47 \mathrm{~g}$.

A fração MFFE-HD (0,53 g) foi refracionada em coluna de vidro $(\phi \times \mathrm{h}=2 \times 12 \mathrm{~cm})$ de sílica gel (230 - 400 mesh) eluída com hex $/ \mathrm{CH}_{2} \mathrm{Cl}_{2}$ (hex: $\mathrm{CH}_{2} \mathrm{Cl}_{2}(7: 3 / 1: 1 / 2: 8) \rightarrow \mathrm{CH}_{2} \mathrm{Cl}_{2}$ ), em gradiente de polaridade crescente. As frações obtidas foram reunidas após análise por CCDA. A fração MFFE-HD7 foi purificada em coluna de gel de Sephadex LH-20 como fase estacionária e eluída com metanol, fornecendo a substância $\mathbf{3}(0,8 \mathrm{mg})$.

A fração MFFE-HA $(0,68 \mathrm{~g})$ foi refracionada em coluna de vidro $(\phi \times \mathrm{h}=2 \times 19 \mathrm{~cm})$ de sílica gel (230 - $400 \mathrm{mesh})$ eluída com $\mathrm{CH}_{2} \mathrm{Cl}_{2} / \mathrm{AcOEt} / \mathrm{MeOH}\left(\mathrm{CH}_{2} \mathrm{Cl}_{2} / \mathrm{AcOEt} 8: 2 \rightarrow \mathrm{MeOH}\right)$. Posteriormente, a fração MFFE-HA4 (124 mg) foi refracionada em coluna de vidro $(\phi \times \mathrm{h}=2,5 \times 11,5 \mathrm{~cm})$ de sílica gel (230 - 400 mesh) eluída com $\mathrm{CH}_{2} \mathrm{Cl}_{2} /$ Acetona $\left(\mathrm{CH}_{2} \mathrm{Cl}_{2} /\right.$ Acetona 9,5:0,5 $\rightarrow$ acetona), fornecendo as substâncias 4 e $5(63 \mathrm{mg})$ em mistura, a qual foi submetida à reação de metilação com diazometano. ${ }^{20}$

\section{Fracionamento da fração $M F F E-A$}

A fração MFFE-A (3,0 g) foi refracionada em coluna sob vácuo, empacotada com sílica gel (70 - 230 mesh) em funil de placa sinterizada $(\phi \times \mathrm{h}=7 \times 26 \mathrm{~cm})$, utilizando-se como fases móveis solventes orgânicos de polaridade crescente: hexano; $\mathrm{CH}_{2} \mathrm{Cl}_{2} ; \mathrm{AcOEt}$ e $\mathrm{MeOH}$. Foram obtidas 4 frações após evaporação dos solventes orgânicos: MFFE-AH $=0,03 \mathrm{~g}$; MFHE-AD $=0,31 \mathrm{~g}$; MFFE-AA $=1,48 \mathrm{~g}$ e MFFE-AM $=0,48 \mathrm{~g}$

A fração MFFE-AD $(0,31 \mathrm{~g})$ foi refracionada em coluna $(\phi \mathrm{x} \mathrm{h}$ $=3 \times 20 \mathrm{~cm}$ ) de sílica gel (230 - 400 mesh), eluída com hex $/ \mathrm{CH}_{2} \mathrm{Cl}_{2}$ $(9,5: 0,5)$ em eluição isocrática. Posteriormente, a fração MFFE-AD4 $(25,4 \mathrm{mg})$ foi refracionada em coluna $(\phi \times \mathrm{h}=1,5 \times 15 \mathrm{~cm})$ de sílica gel (230 - 400 mesh), eluída com $\mathrm{CH}_{2} \mathrm{Cl}_{2} / \operatorname{AcOEt}(9,7: 0,3)$ em eluição isocrática, obtendo-se as substâncias 6 e 7 (6,2 mg) em mistura.
A fração MFFE-AA (1,48 g) foi refracionada em coluna $(\phi \times \mathrm{h}=$ 5 x $14 \mathrm{~cm}$ ) de sílica gel (230 - 400 mesh), eluída com $\mathrm{CH}_{2} \mathrm{Cl}_{2} /$ acetona (7:3) em eluição isocrática. Posteriormente, a fração MFFE-AA3 $(117,6 \mathrm{mg})$ foi refracionada em coluna $(\phi \times \mathrm{h}=3,5 \times 13 \mathrm{~cm})$ de sílica gel (230 - 400 mesh), eluída com $\mathrm{CH}_{2} \mathrm{Cl}_{2} / \mathrm{AcOEt}$ (9:1) em eluição isocrática, obtendo-se novamente a substância $\mathbf{1}$ (14,1 mg). A fração MFFE-AA3.8 (8,2 mg) foi refracionada em coluna de gel de Sephadex $\mathrm{LH}-20(\phi \times \mathrm{h}=2 \times 46 \mathrm{~cm})$ eluída com $\mathrm{MeOH}$ fornecendo novamente a substância $2(1,7 \mathrm{mg})$.

\section{Ensaios biológicos por ingestão com $S$. frugiperda}

Os ensaios biológicos com o extrato bruto e controles foram realizados no Laboratório de Bioensaios do Departamento de Química da UFSCar, mantido a $25 \pm 1{ }^{\circ} \mathrm{C}$, UR de $70 \pm 5 \%$ e fotofase de $12 \mathrm{~h}$.

Uma dieta artificial ${ }^{21}$ contendo água destilada $(600 \mathrm{~mL})$, ágar (10,3 g), extrato de levedura (25,3 g), gérmem de trigo (39,6 g), ácido sórbico (0,8 g), ácido ascórbico (2,6 g), formaldeído 40\% (6,3mL), tetraciclina $(50 \mathrm{mg})$, nipagin $(1,1 \mathrm{~g})$ e feijão carioquinha $(82,5 \mathrm{~g})$ foi utilizada na realização dos ensaios biológicos.

Para a execução dos bioensaios, o extrato etanólico das folhas foi solubilizado em uma pequena quantidade de etanol e misturado ao ácido ascórbico contido na dieta artificial. ${ }^{21}$ Logo após, o solvente foi evaporado e a mistura (ácido ascórbico e extrato) foi incorporada à dieta quando esta atingiu uma temperatura de $50{ }^{\circ} \mathrm{C} .{ }^{22-24}$ Foram utilizadas trinta lagartas para cada tratamento. Além da amostra teste (extrato etanólico incorporado à dieta artificial para S. frugiperda na proporção de $1 \mathrm{~g}$ de extrato para $1 \mathrm{Kg}$ de dieta), foram preparados um tratamento controle (dieta + solvente utilizado para dissolução das amostras) e outro contendo somente com a dieta artificial. Em seguida, os tratamentos foram vertidos em tubos de vidro $(8,5 \times$ $2,5 \mathrm{~cm}$ ), previamente esterilizados, em estufa a $170{ }^{\circ} \mathrm{C}$ por $1 \mathrm{~h} \mathrm{e}$ em seguida tampados com algodão hidrófugo. Após a colocação da dieta, os tubos foram mantidos por $24 \mathrm{~h}$ em grades de arame para eliminação do excesso de umidade (gotículas de água) de suas paredes. A seguir, foi feita a inoculação das lagartas recém-eclodidas de S. frugiperda, utilizando-se uma lagarta por tubo de vidro. As pupas obtidas foram pesadas um dia após a pupação, e transferidas para copos plásticos de $50 \mathrm{~mL}$ de capacidade, onde permaneceram até a emergência dos adultos.

Os parâmetros avaliados foram duração das fases larval e pupal, peso das pupas e porcentagem de insetos mortos (mortalidade) ao final de cada fase. Para cada tratamento foram utilizadas 30 lagartas, distribuídas em três repetições de dez lagartas cada, em delineamento completamente casualizado. Os dados foram submetidos à análise de variância (ANOVA). A comparação entre médias dos tratamentos foi feita através do Teste de Tukey, ao nível de 5\% de probabilidade de erro.

\section{RESULTADOS E DISCUSSÃO}

O estudo químico do extrato etanólico das folhas de $M$. ferruginata resultou no isolamento e identificação dos flavonoides 5,6,7-trihidroxi-4' 'metoxiflavona (1), 5,7,4'-trihidroxi-6,8-dimetilflavona (2), 5-hidroxi-7,4'-dimetoxi-8-metilflavona (3), dos triterpenos ácido ursólico (4) e ácido oleanólico (5) e identificação da mistura dos esteróides $\beta$-sitosterol (6) e estigmasterol (7) (Figura 1). As estruturas dos compostos isolados foram identificadas com base nas análises dos dados de $\mathrm{RMN}{ }^{1} \mathrm{H}$ e ${ }^{13} \mathrm{C} 1 \mathrm{D}$ e $2 \mathrm{D}$ (flavonoides $\mathbf{1}, 2 \mathrm{e}$ 3, Tabela 1), análises através de CG-EM, além da comparação com dados da literatura. ${ }^{25-30}$

As substâncias 1-3 foram isoladas das frações acetato de etila [MFFE-AA3, 1; MFFE-AA3.8, 2], diclorometânica [MFFE-D5.3, 1; 
Tabela 1. Dados de RMN ${ }^{1} \mathrm{H}(400 \mathrm{MHz})$ e ${ }^{13} \mathrm{C}(100 \mathrm{MHz})$ dos compostos 1-3

\begin{tabular}{|c|c|c|c|c|c|c|c|c|c|}
\hline \multirow{2}{*}{$\mathrm{H} / \mathrm{C}$} & \multicolumn{3}{|c|}{ 5,6,7-trihidroxi-4'-metoxiflavona (1) } & \multicolumn{3}{|c|}{ 5,7,4'-trihidroxi-6,8-dimetilflavona (2) } & \multicolumn{3}{|c|}{ 5-hidroxi-7,4'-dimetoxi-8-metilflavona (3) } \\
\hline & $\delta_{\mathrm{H}}{ }^{1}$ & $\delta_{\mathrm{C}}{ }^{1^{*}}$ & HMBC & $\delta_{\mathrm{H}}{ }^{3}$ & $\delta_{\mathrm{C}}{ }^{3 *}$ & HMBC & $\delta_{\mathrm{H}}^{2}$ & $\delta_{\mathrm{C}}{ }^{2 *}$ & HMBC \\
\hline 2 & & 165,0 & & & ND & & & ND & \\
\hline 3 & $6,68(s)$ & 106,6 & $\mathrm{C}-10, \mathrm{C}-1^{\prime}, \mathrm{C}-2, \mathrm{C}-4$ & $6,54(s)$ & 102,1 & $\mathrm{C}-1^{\prime}, \mathrm{C}-4$ & $6,60(\mathrm{~s})$ & 104,7 & $\mathrm{C}-4, \mathrm{C}-10$ \\
\hline 4 & & 184,0 & & & 183,4 & & & 182,6 & \\
\hline 5 & & ND & & & 157,7 & & & 156,2 & \\
\hline 5-OH & $13,14(\mathrm{~s})$ & & & $13,22(\mathrm{~s})$ & & & $12,88(\mathrm{~s})$ & & \\
\hline 6 & & ND & & & 108,8 & & $6,50(\mathrm{~s})$ & 89,5 & C- $10, C-8, C-5$ \\
\hline 7 & & 164,0 & & & 164,9 & & & 163,2 & \\
\hline 8 & $6,79(\mathrm{~s})$ & 92,6 & C-10, C-9, C-7, C-4 & & 103,7 & & & 109,1 & \\
\hline 9 & & 156,0 & & & 154,2 & & & 158,5 & \\
\hline 10 & & 106,0 & & & ND & & & 105,6 & \\
\hline 1 ' & & 123,0 & & & 122,5 & & & ND & \\
\hline 2'/6' & 7,96 (d) & 131,9 & C-4', C-2 & $7,85(\mathrm{~d})$ & ND & C-4' & $7,85(\mathrm{~d})$ & 128,3 & C-2'/6', C-4' \\
\hline $3 ' / 5$ & $7,03(d)$ & 118,9 & $\mathrm{C}-1^{\prime}, \mathrm{C}-4^{\prime}$ & $6,92(d)$ & 115,7 & $\mathrm{C}-1^{\prime}$ & $7,02(d)$ & 114,4 & C-4' \\
\hline 4 ' & & 162,5 & & & 163,1 & & & 162,4 & \\
\hline $7-\mathrm{OCH}_{3}$ & & & & & & & $3,93(\mathrm{~s})$ & 55,7 & C-7 \\
\hline 4'- $-\mathrm{OCH}_{3}$ & 3,98 (s) & 58,6 & & & & & 3,89 (s) & 55,7 & C-4' \\
\hline 6- $\mathrm{CH}_{3}$ & & & & $2,09(\mathrm{~s})$ & ND & C-6, C-5, C-7 & & & \\
\hline $8-\mathrm{CH}_{3}$ & & & & $2,32(\mathrm{~s})$ & 7,9 & C-8, C-9, C-7 & $2,12(\mathrm{~s})$ & 6,94 & C-8, C-7, C-9 \\
\hline
\end{tabular}

${ }^{1}$ Espectros obtidos em $\left(\mathrm{CD}_{3}\right)_{2} \mathrm{CO} ;{ }^{2}$ Espectros obtidos em $\mathrm{CDCl}_{3} ;{ }^{3}$ Espectos obtidos em MeOD;* valores obtidos através das projeções de carbono do HSQC e HMBC; ND: não determinado.

MFFE-D5.7, 2], e hexânica [MFFE-HD7, 3], todas da partição do extrato etanólico das folhas de $M$. ferruginata.

Nos espectros de RMN de ${ }^{1} \mathrm{H}$ de $\mathbf{1 - 3}$ foram observadas as presenças de dois dubletos, em um sistema AA'BB' de acoplamento de spin, na região de hidrogênios ligados a carbonos aromáticos em $\delta_{\mathrm{H}}$ 7,03 e 7,96 (1), 6,92 e 7,85 (2) e 7,02 e 7,85 (3), integrados para dois hidrogênios cada, com constante de acoplamento de $8,4 \mathrm{~Hz}$, atribuídos à H-3'/5' e H-2'/6', respectivamente, caracterizando o anel B de flavona para estes compostos. Nestes mesmos experimentos foram observados também: para 1 três singletos em $\delta_{\mathrm{H}} 6,79(\mathrm{H}-8), 6,68(\mathrm{H}-$ 3) e 3,98 $\left(\mathrm{OCH}_{3}-4^{\prime}\right)$, integrando respectivamente para um, um e três hidrogênios, sendo o último sinal referente aos hidrogênios metílicos<smiles>[R9]c1c([R3])c(O)c2c(=O)cc(-c3ccc(O)cc3)oc2c1[R]</smiles>

$1 \mathrm{R}=\mathrm{CH}_{3}, \mathrm{R}_{1}=\mathrm{R}_{2}=\mathrm{H}, \mathrm{R}_{3}=\mathrm{OH}$

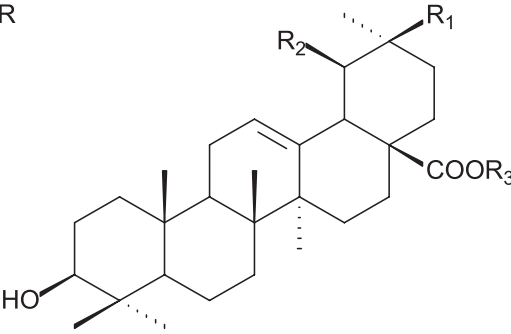

$$
\begin{array}{ll}
2 \mathrm{R}=\mathrm{R}_{1}=\mathrm{H}, \mathrm{R}_{2}=\mathrm{R}_{3}=\mathrm{CH}_{3} & 4 \mathrm{R}_{1}=\mathrm{R}_{3}=\mathrm{H}, \mathrm{R}_{2}=\mathrm{CH}_{3} ; 8 \mathrm{R}=\mathrm{H}, \mathrm{R}_{2}=\mathrm{R}_{3}=\mathrm{CH}_{3} \\
3 \mathrm{R}=\mathrm{R}_{1}=\mathrm{R}_{2}=\mathrm{CH}_{3}, \mathrm{R}_{3}=\mathrm{H} & 5 \mathrm{R}_{1}=\mathrm{CH}_{3}, \mathrm{R}_{2}=\mathrm{R}_{3}=\mathrm{H} ; 9 \mathrm{R}_{1}=\mathrm{R}_{3}=\mathrm{CH}_{3}, \mathrm{R}_{2}=\mathrm{H}
\end{array}
$$<smiles>CCC(/C=C/C(C)C1CCC2C1CCC1(C)C2CCC2(C)C3(C)CCC(O)CC3=CCC12C)C(C)C</smiles>

Figura 1. Estruturas dos compostos 1-9 
ligados a um heteroátomo; um singleto em $\delta_{\mathrm{H}} 13,14$, característico de flavonoides contendo um grupo hidroxila na posição C-5, sugerindo ser uma flavona contendo um substituinte na posição C-4' e o anel A tri-substituído; ${ }^{28}$ para 2 observou-se um singleto em $\delta_{\mathrm{H}} 6,54(\mathrm{H}-3)$ integrando para um hidrogênio e dois singletos intensos de hidrogênios metílicos em $\delta_{\mathrm{H}} 2,32\left(\mathrm{CH}_{3}-8\right)$ e $\delta_{\mathrm{H}} 2,09\left(\mathrm{CH}_{3}-6\right)$ integrando para três hidrogênios cada; estas observações sugeriram a presença de uma flavona contendo um substituinte na posição C-4' e um anel A completamente substituído. Para 3 foram observados dois singletos em $\delta_{\mathrm{H}} 6,60(\mathrm{H}-3)$ e $6,50(\mathrm{H}-6)$ integrados para um hidrogênio cada e dois singletos em $\delta_{\mathrm{H}} 3,93\left(7-\mathrm{OCH}_{3}\right)$ e $3,89\left(4^{\prime}-\mathrm{OCH}_{3}\right)$ referentes aos hidrogênios metílicos das metoxilas aromáticas e um singleto intenso de hidrogênio metílico em $\delta_{\mathrm{H}} 2,12\left(8-\mathrm{CH}_{3}\right)$, integrando para três hidrogênios e um singleto em $\delta_{\mathrm{H}} 12,88(5-\mathrm{OH})$, característico de flavonoides contendo um grupo hidroxila na posição C-5.

Nos mapas de contornos de HSQC pôde-se observar que os singletos em $\delta_{\mathrm{H}} 6,68(\mathbf{1}), 6,54(\mathbf{2})$ e 6,60 (3) apresentavam correlações com os sinais de carbonos em $\delta_{\mathrm{C}} 106,6$ (1), 102,1 (2) e 104,7 (3) característicos dos hidrogênios $\mathrm{H}-3$ ligados aos $\mathrm{C}-3$ de uma flavona. Nos experimentos de HMBC observou-se as correlações destes singletos com os carbonos em $\delta_{\mathrm{C}} 106,0 ; 123,0 ; 165,0$ e 184,0 atribuídos aos carbonos C-10, C-1', C-2 e C-4 para 1; $\delta_{\mathrm{C}} 122,5$ e 183,4 atribuídos aos carbonos C-1' e C-4 para 2 e $\delta_{\mathrm{C}} 105,6$ e 182,6 atribuídos aos carbonos $\mathrm{C}-10$ e $\mathrm{C}-4$ para $\mathbf{3}$, respectivamente. No mapa de contorno de HMBC de 1 , as correlações dos singletos em $\delta_{\mathrm{H}} 6,79(\mathrm{H}-8)$ com os sinais de carbonos em $\delta_{\mathrm{C}} 106,0 ; 156,0 ; 164,0$ e 184,0 atribuídos aos carbonos C-10, C-9, C-7 e C-4 para 1, sugeriram a ligação deste hidrogênio ao carbono C-8.

Os dubletos em $\delta_{\mathrm{H}}$ 7,03 (1), 6,92 (2) e 7,02 (3) (H-3'/5') mostraram correlações com os sinais de carbonos em $\delta_{\mathrm{C}} 118,9 ; 115,7$ e 114,4 , respectivamente, referentes aos carbonos C-3'/5', enquanto nos experimentos de HMBC foram observadas as correlações destes hidrogênios com os carbonos em $\delta_{\mathrm{C}} 123,0$ e 162,5 (1), 122,5 e 163,1 (2) e 162,4 (3), respectivamente, referentes aos carbonos C-1' e C-4'. Nos experimentos de HSQC observou-se as correlações dos dubletos em $\delta_{\mathrm{H}} 7,96(\mathbf{1})$ e 7,85 (3) (H-2'/6') com os sinais de carbonos em $\delta_{\mathrm{C}}$ $131,9(\mathbf{1}), 128,3$ (3), referentes aos carbonos C-2'/6'. Nos experimentos de HMBC pôde-se observar as correlações destes hidrogênios $\left(\mathrm{H}^{2}{ }^{\prime} / 6^{\prime}\right)$ com os sinais de carbono em $\delta_{\mathrm{C}} 162,5$ (C-4') e $\delta_{\mathrm{C}} 165,0$ (C-2) para 1, 163,1 (C-4') para 2 e 162,4 (C-4') para 3.

$\mathrm{O}$ hidrogênio metílico em $\delta_{\mathrm{H}} 2,32\left(8-\mathrm{CH}_{3}\right)$ de 2 mostrou correlação, no experimento de HSQC, com o sinal de carbono em $\delta_{\mathrm{C}} 7,9$, correspondente ao carbono da metila ligada ao C-8. No mapa de contorno HMBC este hidrogênio correlacionou-se com os sinais de carbono em $\delta_{\mathrm{C}} 103,7 ; \delta_{\mathrm{C}} 154,2$ e $\delta_{\mathrm{C}} 164,9$; referentes aos carbonos C-8, C-9 e C-7, respectivamente. O segundo sinal de hidrogênio metílico de 2, com deslocamento $\delta_{\mathrm{H}} 2,09\left(6-\mathrm{CH}_{3}\right)$ mostrou correlação com sinais de carbono em $\delta_{\mathrm{C}} 108,8, \delta_{\mathrm{C}} 157,7$ e $\delta_{\mathrm{C}} 164,9$, referentes aos carbonos C-6, C-5 e C-7, respectivamente, o que confirmou a ligação desta metila ao carbono $C-6$. A presença de uma hidroxila $\left(\delta_{\mathrm{H}} 13,22\right)$ em C-5 em 2 foi confirmada com a obtenção do espectro em acetona deuterada, análise dos dados e comparação permitiram identificá-la como a flavona 5,7,4'-trihidroxi-6,8-dimetilflavona (2). ${ }^{26}$

Nos mapas de contornos de HSQC pôde-se observar que os singletos em $\delta_{\mathrm{H}} 3,98\left(4^{\prime}-\mathrm{OCH}_{3}\right)$ para $1, \delta_{\mathrm{H}} 3,93\left(7-\mathrm{OCH}_{3}\right)$ e 3,89 $\left(4^{\prime}-\mathrm{OCH}_{3}\right)$ para 3 correlacionam com os sinais de carbonos em $\delta_{\mathrm{C}}$ 58,6 (1) e 55,7 (3) e para 3 um único sinal para 2 carbonos indicou que as metoxilas não estavam orto dissubstituídas. Nos experimentos de $\mathrm{HMBC}$ os singletos das metoxilas em $\delta_{\mathrm{H}} 3,98(\mathbf{1}), 3,89$ e 3,93(3) mostraram correlações com os sinais de carbonos em 162,5 e 162,4, atribuídos aos carbonos C-4' e $\delta_{\mathrm{C}} 163,2$ atribuído a carbono C-7.

No HSQC de 3 o sinal do hidrogênio metílico $\left(8-\mathrm{CH}_{3}\right)$ em $\delta_{\mathrm{H}} 2,12$ apresentou correlação com um sinal de carbono em $\delta_{\mathrm{C}} 6,9$, referente ao carbono $\mathrm{CH}_{3}-8$. No mapa de contorno de $\mathrm{HMBC}$ foi possível observar, além das correlações com os carbonos C-8 $\left(\delta_{\mathrm{C}} 109,1\right)$ e C-7 $\left(\delta_{\mathrm{C}} 163,2\right)$, correlação com um sinal de carbono em $\delta_{\mathrm{C}} 158,5$ atribuído ao carbono C-9, confirmando a ligação da metila ao C-8.

A ocorrência natural da 5-hidroxi-7,4'-dimetoxi-8-metilflavona (3) foi proposta pela primeira vez em um trabalho com as folhas de Eucalyptus camaldulensis. ${ }^{27}$

A $C$-alquilação dos flavonoides pode acontecer de duas formas distintas; a primeira se dá através da ligação de um açúcar diretamente ao núcleo flavonoídico, enquanto a segunda envolve a ligação de diferentes grupos alquila, geralmente do grupo metila, a um dos carbonos do flavonoide. Os primeiros trabalhos envolvendo o isolamento de flavonoides $C$-metilados se referem a espécies de Pinus (Pinaceae) e à Lonchitis tisserantii, ${ }^{31}$ nas quais se encontrou a 6-C-metilcrisina; às espécies do gênero Matteuccia, das quais foi isolada a flavona $6,8-d i-C$-metilada matteucinol ${ }^{32} \mathrm{e}$ às espécies de Eucalyptus, onde foram encontrados a eucaliptina e a sideroxilina. ${ }^{33}$ As famílias que mais contribuíram com esse tipo de flavonoide foram Myrtaceae e Ericaceae. Podem também ser citadas as famílias Pteridaceae, Pinaceae, Agavaceae, Liliaceae, Annonaceae, Clusiaceae, Didieriaceae, Nyctaginaceae, Fabaceae, Meliaceae, Platanaceae e Asteraceae. Na maioria destas famílias, com exceção de Myrtaceae e Ericaceae, os flavonoides $C$-metilados geralmente são encontrados em apenas um gênero e num reduzido número de espécies. ${ }^{34}$ Até onde se conhece, esta é a primeira vez que é relatada a presença de flavonoides C-metilados no gênero Miconia e, provavelmente, na família Melastomataceae. Dos flavonoides $C$-metilados, nenhum apresentou $C$-metilação no anel B. ${ }^{35,36} \mathrm{~A}$ metilação nos carbonos C-6 e C-8, e em ambas as posições, são mais comuns, enquanto que a metilação no carbono C-3 é de rara ocorrência. As substâncias $\mathbf{2}$ e $\mathbf{3}$ tiveram suas fórmulas moleculares confirmadas através dos espectros de massa de alta resolução (EMAR), $\mathrm{C}_{17} \mathrm{H}_{14} \mathrm{O}_{5}$ (298,0841 DA) e $\mathrm{C}_{16} \mathrm{H}_{12} \mathrm{O}_{5}$ (284,0684 DA) respectivamente.

As substâncias $\mathbf{4}$ e $\mathbf{5}$ foram isoladas do extrato etanólico, fração hexânica, de folhas de M. ferruginata. Foram observados sinais característicos de triterpenos no espectro de $\mathrm{RMN}{ }^{1} \mathrm{H}$, sendo a amostra submetida à reação de metilação com diazometano, a fim de ser analisada por CG-EM. O espectro de $\mathrm{RMN}{ }^{1} \mathrm{H}$ da amostra metilada mostrou sinais centrados em $\delta_{\mathrm{H}} 5,22$ e 5,21, referentes aos H-12 olefínicos característicos dos esqueletos ursânicos e oleanânicos, sugerindo que a amostra se tratava de uma mistura destes triterpenos. Os sinais referentes aos $\mathrm{H}-3$ carbinólicos apareceram centrados em $\delta_{\mathrm{H}} 3,20$ e $3,16(\mathrm{~m})$, e os sinais referentes aos $\mathrm{H}$ metílicos entre $\delta_{\mathrm{H}} 1,10-0,69$. Foi observada ainda a presença de dois singletos em $\delta_{\mathrm{H}} 3,59$ e 3,57; relativos aos hidrogênios metoxílicos. O mapa de contorno de HSQC mostrou a correlação do hidrogênio em $\delta_{\mathrm{H}} 5,22$ com o sinal de carbono em $\delta_{\mathrm{C}} 122,6$ referente ao carbono vinílico C-12 do esqueleto oleanânico. O sinal em $\delta_{\mathrm{H}} 5,21$ correlacionou com o carbono em $\delta_{\mathrm{C}} 125,8$ característico do C-12 do esqueleto ursânico. A presença do carbono carbinólico foi observada com deslocamento químico em $\delta_{\mathrm{C}} 79,3$ característico da configuração $\beta$ em torno do $\mathrm{C}-3,{ }^{37}$ e a presença de carbonila típica de éster metílico C-28, em $\delta_{\mathrm{C}}$ 178,0. A partir desses dados e pelos sinais de carbono projetados no HMBC pôde-se concluir que a substância 4 era o ácido ursólico em mistura com a substância 5, ácido oleanólico, presente em menor quantidade. A reação de metilação da amostra levou à formação dos ésteres ursolato e oleanolato de metila, 8 e 9, respectivamente, confirmados por comparação dos espectros de massas. ${ }^{38}$

As substâncias 6 e 7 foram obtidas da fração acetato de etila (MFFE-AD). A identificação destes esteróides foi realizada através de dados de RMN ${ }^{1} \mathrm{H}$ e CG-EM. No espectro de RMN ${ }^{1} \mathrm{H}$ observou-se a presença de um dubleto largo em $\delta_{\mathrm{H}} 5,34$ (H-6), de sinais de hidrogênios vinílicos em $\delta_{\mathrm{H}} 5,14$ (dd, $J=8,9$ e $\left.15 \mathrm{~Hz}\right), \delta_{\mathrm{H}} 5,07$ (dd, 
Tabela 2. Médias da duração da fase larval e pupal, peso pupal, mortalidade da fase larval de $S$. frugiperda alimentada com dieta artificial tratada com o extrato etanólico das folhas, a $1000 \mathrm{mg} \mathrm{kg}^{-1}$, de M. ferruginata. Temp.: $25 \pm 1{ }^{\circ} \mathrm{C}$; UR: $70 \pm 5 \%$ e fotofase: $12 \mathrm{~h}$

\begin{tabular}{lcccc}
\hline Tratamento & $\begin{array}{c}\text { Duração da fase larval (dias) } \\
( \pm \mathrm{EP})^{1}\end{array}$ & $\begin{array}{c}\text { Duração da fase pupal (dias) } \\
( \pm \mathrm{EP})^{1}\end{array}$ & $\begin{array}{c}\text { Peso pupal }(\mathrm{mg})( \pm \mathrm{EP})^{1} \\
\text { Mortalidade larval }(\%)( \pm \mathrm{EP})^{1}\end{array}$ \\
\hline M. ferruginata & $34,00 \pm 5,02 \mathrm{a}$ & $18,61 \pm 1,12 \mathrm{a}$ & $271,38 \pm 19,74 \mathrm{a}$ & $56,67 \pm 20,82 \mathrm{a}$ \\
Controle (Dieta + solvente) & $21,36 \pm 1,62 \mathrm{~b}$ & $10,80 \pm 1,20 \mathrm{~b}$ & $266,95 \pm 27,18 \mathrm{a}$ & $16,67 \pm 5,77 \mathrm{~b}$ \\
Dieta & $17,44 \pm 2,41 \mathrm{~b}$ & $10,12 \pm 1,54 \mathrm{~b}$ & $271,63 \pm 27,34 \mathrm{a}$ & $10,00 \pm 0,00 \mathrm{~b}$ \\
\hline
\end{tabular}

${ }^{1}$ Médias seguidas da mesma letra na coluna, não diferem entre si pelo Teste de Tukey $(\mathrm{P} \leq 0,05)$.

$J=8,9$ e $15 \mathrm{~Hz}$ ) atribuídos aos hidrogênios H-22 e H-23 da cadeia lateral do estigmasterol; um multipleto em $\delta_{\mathrm{H}} 3,55$ característico dos hidrogênios H-3 dos esteróides sitosterol e estigmasterol. Pôde-se verificar ainda a presença de um grande número de sinais referentes a hidrogênios metílicos, metilênicos e metínicos na região entre $\delta_{\mathrm{H}} 0,68-2,41$ característicos do esqueleto esteroidal. Para a confirmação dos compostos presentes na mistura foi realizada análise por CG-EM. As estruturas destas substâncias foram confirmadas através dos picos referentes aos íons moleculares $\operatorname{com} \mathrm{m} / \mathrm{z} 414 \mathrm{e} 412$, os quais apresentam as massas moleculares esperadas $\mathrm{C}_{29} \mathrm{H}_{50} \mathrm{O}$ e $\mathrm{C}_{29} \mathrm{H}_{48} \mathrm{O}$ para sitosterol (6) e estigmasterol (7), respectivamente e pelo padrão de fragmentação comparado com a biblioteca do equipamento de espectrometria de massas.

\section{Ensaios biológicos por ingestão com $S$. frugiperda}

Ao compararmos as lagartas alimentadas com o extrato etanólico das folhas de $M$. ferruginata incorporado à dieta artificial com os grupos do controle (dieta + solvente) e da dieta, observou-se diferenças para a duração da fase larval, pupal e mortalidade larval (Tabela 2). As lagartas alimentadas com o extrato etanólico das folhas de $M$. ferruginata apresentaram um alongamento da fase larval de 12,64 dias quando comparadas as lagartas alimentadas somente com a dieta artificial e de 16,56 dias para as lagartas alimentas com o controle (Tabela 2). A fase pupal também é afetada, apresentando alongamento de 8,49 e 7,81 dias em relação ao controle e a dieta artificial, respectivamente. Experimentos realizados com extratos de Azadirachtina indica (Meliaceae) sobre S. frugiperda, observaram um aumento da duração fase larval em 10,1 dias. ${ }^{39} \mathrm{O}$ aumento da duração da fase larval mantém a lagarta mais suscetível a ataques de predadores, parasitas, parasitóides, entomopatógenos, intempéries climáticas e ainda com maior competição por alimento. ${ }^{40}$ Segundo Martinez e Emden ${ }^{41}$ a inibição do crescimento é função da reduzida ingestão de alimentos, pouca habilidade de conversão de nutrientes, enquanto o alongamento da duração da fase larval se verifica em geral pela reduzida ingestão de alimentos em razão da existência de um inibidor ou vários inibidores nos alimentos, ou uma inadequação nutricional do substrato alimentar. Os adultos que emergirão desses indivíduos terão assincronia com a população normal e, consequentemente, a cópula será dificultada ou quando existir tenderá à consanguinidade pelo acasalamento de indivíduos da mesma geração. Também será diminuído o número de gerações do inseto no ciclo agrícola. ${ }^{42}$ Sugere-se, portanto, haver a presença de substâncias capazes de diminuir ou inibir a alimentação das lagartas presentes no extrato de folhas de $M$. ferruginata.

Para o tratamento em que as lagartas foram alimentadas com o extrato etanólico das folhas de $M$. ferruginata na concentração de $1000 \mathrm{mg} \mathrm{mL}^{-1}$ foi observado uma mortalidade larval de 56,67\% quando comparado ao controle. A redução do crescimento aliada à mortalidade faz com que a lagarta apresente um alto gasto energético para quebrar substâncias tóxicas presentes em seu organismo ao invés de ter esse custo energético direcionado para o crescimento e pupação.
Seu potencial tem efeitos principalmente sobre a duração e mortalidade da fase larval. ${ }^{42}$ Os resultados apresentados para M. ferruginata sugerem eficiência deste extrato como inseticida, pois ocasiona efeito na lagarta logo nas primeiras fases de desenvolvimento, o que poderia reduzir os prejuízos na produção agrícola. Os resultados obtidos sugerem que novos trabalhos com outras partes vegetais e substâncias isoladas para que se possa conhecer melhor a sua eficácia.

\section{CONCLUSÃO}

Este estudo constitui o primeiro relato na literatura do efeito inseticida do extrato etanólico de folhas de $M$. ferruginata. Além disso, foram isolados os flavonoides: 5,6,7-trihidroxi-4'-metoxiflavona, 5-hidroxi-7,4'-dimetoxi-8-metilflavona, 5,7,4'-trihidroxi-6,8-dimetilflavona, dois triterpenos: ácidos ursólico e oleanólico e a miustura dos esteroides: $\beta$-sitosterol e estigmasterol. O extrato etanólico das folhas de $M$. ferruginata apresentou efeito inseticida sobre as lagartas de $S$. frugiperda. Estes resultados sugerem a realização de novos estudos com os extratos de outras partes vegetais e com as substâncias isoladas.

\section{MATERIAL SUPLEMENTAR}

Todos os espectros de RMN, mapas de contorno HSQC, HMBC, EMAR estão disponíveis em http://quimicanova.sbq.org.br, em arquivo pdf, com livre acesso.

\section{AGRADECIMENTOS}

Os autores agradecem ao CNPq (Conselho Nacional de Desenvolvimento Científico e Tecnológico), CAPES (Coordenação de Aperfeiçoamento de Pessoal de Nível Superior) e FAPESP (Fundação de Amparo à Pesquisa do Estado de São Paulo, proc. 2012/252996) pelas bolsas e apoios financeiros concedidos e ao LACEM, Laboratório de Espectrometria de Massas da Universidade Federal de Goiás pelos espectros de massas de alta resolução.

\section{REFERÊNCIAS}

1. Martins, A. B. Em Flora Fanerogâmica do Estado de São Paulo; Marins, S. E., Wanderley, M. G. L., Shepherd, G. J., Giulietti, A. M., Melhem, T. S., eds.; Instituto de Botânica: São Paulo, 2009, vol. 6.

2. Meyer, J. Y.; Biotropica 1998, 30, 609.

3. Martins, A. B.; Semir, J.; Goldenberg, R.; Martins, E.; Acta Bot. Bras. 1996, 10, 267.

4. Gunatilaka, A. A. L.; Berger, J. M.; Evans, R., Miller, J. S.; Wisse, J. H.; Neddermann, K. M.; Bursuker, I.; Kingston, D. G. I.; J. Nat. Prod. 2001, 64,2 .

5. Chan, W. R.; Sheppard, V.; Medford, K. A.; Tinto, W. F.; Reynolds, W. F.; McLean, S.; J. Nat. Prod. 1992, 55, 963.

6. Spessoto, M. A.; Ferreira, D. S.; Crotti, A. E. M.; Silva, M. L. A.; Cunha, W. R.; Phytomedicine 2003, 10, 606. 
7. Cunha, W. R.; Crevelin, E. J.; Arantes, G. M.; Crotti, A. E. M.; Andrade, E.; Silva, M. L.; Furtado, N. A. J. C.; Albuquerque, S.; Ferreira, D. S.; Phytother. Res. 2006, 20, 474.

8. Cunha, W. R.; De Matos, G. X.; Souza, M. G.; Tozatti, M. G.; Andrade e Silva, M. L.; Martins, C. H.; Da Silva, R.; Da Silva Filho, A. A.; Pharm. Biol. 2010, 48, 166.

9. Peixoto, J. A.; Andrade e Silva, M. L.; Crotti, A. E. M.; Veneziani, R. C. S.; Gimenez, V. M. M.; Januário, A. H.; Groppo, M.; Magalhães, L. G.; Dos Santos, F. F.; Albuquerque, S.; Da Silva Filho, A. A.; Cunha, W. R.; Molecules 2011, 16, 1825.

10. Rodrigues, J.; Da Silva, M. A.; Dos Santos, L. C.; Vilegas, W.; Resumos da $30^{a}$ Reunião Anual da Sociedade Brasileira de Química, Águas de Lindóia, Brasil, 2007.

11. Rodrigues, J.; Rinaldo, D.; Dos Santos, L. C.; Vilegas, W.; Phytochemistry 2007, 68, 1781.

12. Mancini, E.; De Martino, L.; Belisario, M. A.; De Feo, V.; Pharmacologyonline 2008, 2, 452.

13. Serpeloni, J. M.; Vilegas, W.; Varanda, E. A.; Cólus, I. M.; Semina: Ciências Biológicas e da Saúde 2008, 29, 47.

14. Lima, L. A. R.; Dos Santos, D. L.; Ferreira, H. D.; Tresvenzol, L. M. F.; De Paula, J. R.; Fiuza, T. S.; Revista de Biotecnologia \& Ciência 2013, 2, 1037.

15. Barroso, P. R.; Otoni, T. J. O.; Mendes, J. P. G.; Machado, E. L. M.; Martins, H. R.; Gregorio, L. E.; Chem. Nat. Compd. 2017, 53, 167.

16. Rodríguez, J.; Montoya-Lerma, J.; Calle, Z.; J. Insect Sci. 2015, 15, 1.

17. Guldbrandsen, N.; De Mieri, M.; Mgupta, M.; Seiser, T.; Wiebe, C.; Dickhaut, J.; Reingruber, R.; Sorgenfrei, O.; Hamburguer, M.; Sci Pharm. 2015, 83, 353.

18. Cruz, I. A lagarta-do-cartucho na cultura do milho. EMBRAPACNPMS: Sete Lagoas, 1995.

19. Jesus, F. L.; Boiçá Junior, A. L.; Em Tópicos em Entomologia Agrícola II; Silva, A. G., Rodrigues, C. A., Becaro, C. K., Bottega, D. B., Haddad, G. K., Alvez, G. C. S., Janini, J. C., eds.; Funep: Jaboticabal, 2009.

20. Leonard, J.; Lygo, B.; Procter, G.; Advanced Practical Organic Chemistry, $2^{\text {th }}$ ed., Blackie Academic \& Professional: London, 1995.

21. Kasten, P. J. R.; Precetti, A. A. C. M.; Parra, J. R. P.; Rev. Agric. 1978, 53,68 .

22. Matos, A. P.; Nebo, L.; Calegari, E. R.; Batista-Pereira, L. G.; Vieira, P. C.; Fernandes, J. B.; Da Silva, M. F. G. F.; Ferreira-Filho, P.; Rodriguez, R. R.; Bioassay 2006, $1,1$.
23. Matos, A. P.; Leite, A. C.; Batista-Pereira, L. G.; Vieira, P. C.; Fernandes, J. B.; Da Silva, M. F. G. F.; Z. Naturforsch., C: J. Biosci. 2009, 64, 441.

24. Leite, A. C.; Matos, A. P.; Batista-Pereira, L. G.; Fernandes, J. B.; Vieira, P. C.; Da Silva, M. F. G. F.; Biopest. Int. 2008, 4, 28.

25. Horie, T.; Ohtsuru, Y.; Shibata, K.; Yamashita, K.; Tsukayama, M.; Kawamura, Y.; Phytochemistry 1998, 47, 865.

26. Youssef, D. T. A.; Ramadan, M. A.; Khalifa, A. A.; Phytochemistry 1998, 49, 2579.

27. El-Ghorab, A. H.; El-Massry, K. F.; Marx, F.; Fadel, H. M.; Nahrung 2003, 47,41 .

28. Gao, H.; Kawabata, J.; Biosci. Biotechnol. Biochem. 2004, 68, 1858.

29. Kang, S.; Li, Y. W. L.; Zheng, S.; Indian J. Chem. 2004, 43B, 1332.

30. Goulart, M. O. I.; Sant'Ana, A. E. G.; Lima, R. A.; Cavalcante, S. H.; Carvalho, M. G.; Braz-Filho, R.; Quim. Nova 1993, 16, 95.

31. Lebreton, P.; Jay, M.; Voirin, B.; Chim. Anal. 1967, 49, 375.

32. Harborne, J. B.; Comparative Biochemistry of the Flavonoids, Academic Press: London, New York, 1967.

33. Wollenweber, E.; Kohorst, G.; Z. Naturforsch., C: J. Biosci. 1981, 36, 913.

34. Bohm, B. A.; Introduction to flavonoids. Harwood Academic Publishers: Amsterdam, 1998.

35. Wollenweber, E.; Jay, M.; Em The flavonoids advances in research since 1980; Harborne, J. B., ed.; ${ }^{\text {st }}$ ed., Chapman and Hall: London, 1988, cap. 7.

36. Wollenweber, E.; Em The Flavonoids advances in research since 1986; Harborne, J. B., ed., Chapman and Hall: London, 1994.

37. Junges, M. J.; Fernandes, J. B.; Vieira, P. C.; Fernandes, M. F. G. S.; Filho, E. R.; Fruhauf, M.; Barañano, A. G.; Revista Pesquisa e Pósgraduação 2000, 1, 13.

38. Piozzi, F.; Paternostro, M.; Passannanti, S.; Gacs-Baitz, E.; Phytochemistry 1986, 25, 539.

39. Forim, M. R.; Matos, A. P.; Silva, M. F. G. F.; Cass, Q. B.; Vieira, P. C.; Fernandes, J. B.; Quim. Nova 2010, 33, 1082.

40. Rodríguez, H. C.; Vendramin, J. D.; Manejo Integrado de Plagas 1996, $42,14$.

41. Martinez, S. S.; Emden, H. F. V.; Neotrop. Entomol. 2001, 30, 113.

42. Tanzubil, P. B.; McCaffery, A. R.; Crop Prot. 1990, 9, 383. 